\title{
LÓGICA NEBULOSA APLICADA À PROTEÇÃO DIFERENCIAL DE TRANSFORMADORES DE POTÊNCIA
}

\author{
Daniel Barbosa* \\ daniel. barbosa@pro.unifacs.br \\ Denis V. Coury ${ }^{\dagger}$ \\ coury@sc.usp.br
}

\author{
Ulisses Chemin Netto ${ }^{\dagger}$ \\ uchenin@usp.br \\ Mário Oleskovicz ${ }^{\dagger}$ \\ olesk@sc.usp.br
}

\begin{abstract}
${ }^{*}$ Universidade Salvador, Mestrado em Energia, Rua Ponciano de Oliveira, 126, $2^{\circ}$ andar, Rio Vermelho, 41.950-275, Salvador, BA

${ }^{\dagger}$ Universidade de São Paulo - Escola de Engenharia de São Carlos, Departamento de Engenharia Elétrica, Av. Trabalhador São Carlense, 400, Centro, 13.566-590, São Carlos, SP
\end{abstract}

\begin{abstract}
Fuzzy Logic Applied to Power Transformer Differential Protection

This article presents a new algorithm based on fuzzy logic for differential protection of power transformers. The fuzzy approach used in this work is able to detect and discriminate between an internal fault and other operating conditions, such as energization, sympathetic inrush and overexcitation. The electrical power system, including the power transformer studied, was modeled using the ATP (Alternative Transients Program) software to obtain data of operational conditions and fault situations in order to test the developed algorithm. Concerning the validation process, the results were compared to a commercial relay, showing the advantages of the proposed method.
\end{abstract}

KEYWORDS: Electrical power system, Differential protection, Power transformer, Sympathetic inrush, Fuzzy logic, ATP.
Artigo submetido em 29/05/2010 (Id.: 01156)

Revisado em 27/10/2010, 14/01/2011, 15/04/2011

Aceito sob recomendação do Editor Associado Prof. Takashi Yoneyama

\section{RESUMO}

Este artigo apresenta um algoritmo baseado na lógica nebulosa para a proteção diferencial de transformadores de potência. A abordagem nebulosa utilizada neste trabalho permite detectar os defeitos internos, distinguindo-os de outras situações operativas, como a energização, a energização solidária e a sobre-excitação. O sistema elétrico de potência, incluindo o transformador estudado, foi modelado utilizando-se do software ATP (Alternative Transients Program) para gerar dados de situações de operação e falta, as quais são empregadas durante a etapa de testes do algoritmo proposto. Cabe comentar que na validação, os resultados foram comparados com um relé digital comercial, mostrando as vantagens do método proposto.

PALAVRAS-CHAVE: Sistema elétrico de potência, Proteção diferencial, Transformador de potência, Energização solidária, Lógica nebulosa, ATP.

\section{INTRODUÇÃO}

Os transformadores de potência são equipamentos essenciais para a operação e a integração do sistema elétrico. Tal condição implica na necessidade de sistemas de monitoramento e proteção eficientes. Estes devem 
ser capazes de detectar as ocorrências de defeitos e/ou condições anormais que podem comprometer o seu adequado funcionamento ou a continuidade no fornecimento da energia elétrica.

Os esquemas de proteção aplicados nestas máquinas podem ser agrupados em dois grandes tópicos: as proteções intrínsecas (pertencentes à construção do transformador) e extrínsecas (não pertencentes à construção do transformador). Dentre os exemplos que compõem o primeiro grupo estão o relé Buchholz (ANSI 63), a válvula de alívio de pressão (ANSI 63S) e o relé de imagem térmica (ANSI 49). Todos esses são acessórios, em geral, adquiridos na compra do transformador, sendo o princípio de funcionamento baseado em uma grandeza de origem mecânica. O segundo grupo é, geralmente, composto por equipamentos que baseiam seu princípio de funcionamento em grandezas de natureza elétrica, como os fusíveis de potência, as funções de sobrecorrente (ANSI 50/51), a função diferencial de corrente (ANSI 87) e a função de sobre-excitação (ANSI 24) (IEEE Std. C37.91, 2008).

Dentre as diversas proteções de natureza elétrica aplicáveis aos transformadores de potência, a função diferencial percentual de corrente (ANSI 87T) é a mais utilizada, uma vez que permite a discriminação entre faltas internas de outras condições operativas, além da tomada de decisão de abertura de um disjuntor ou disjuntores associados (Blackburn e Domin, 2007). Embora esta técnica possa ser utilizada em qualquer transformador, sua aplicação é comumente observada em transformadores com potência nominal próxima ou superior a 10MVA (IEEE Std. C37.91, 2008).

Normalmente, os relés diferenciais percentuais baseiamse no princípio da comparação das correntes dos terminais do equipamento protegido com limiares prédeterminados. Assim, quando existe uma ocorrência de defeito interno, os disjuntores associados são acionados e o equipamento é desconectado do sistema de suprimento. Todavia, tal procedimento pode ser indevidamente sensibilizado por algumas situações de operação, oferecendo, então, algumas limitações em sua aplicação. Dentre as condições que podem provocar a atuação indevida, tem-se, por exemplo, a energização, a sobreexcitação e a energização solidária (sympathetic inrush) (Sengül et al., 2005).

Para transpor tais limitações, diversas soluções são encontradas na literatura, dentre as quais destaca-se a utilização de componentes harmônicas, uma vez que cada fenômeno é caracterizado por um determinado espectro de frequência. Como exemplo, é possível citar a presença da componente de segunda harmônica durante a corrente de energização, e a componente de quinta harmônica durante a sobre-excitação (Tripathy et al., 2006). Contudo, tal mecanismo não consegue contemplar todas as situações operativas existentes, como por exemplo, a energização solidária. Isso implica na possibilidade de eventuais falhas nos sistemas de proteção, que se baseiam nas restrições harmônicas.

Desta forma, engenheiros e pesquisadores buscam continuamente inovações que melhorem a precisão e a estabilidade dos algoritmos aplicados nos relés de proteção diferencial na discriminação das condições operativas supracitadas. Dentre as diversas técnicas propostas, Tripathy et al. (2010) e Perez et al. (1994) propuseram algoritmos baseados em redes neurais artificiais (RNAs). Em 2008, Megahed et al., reportaram um algoritmo utilizando a transformada Wavelet para a proteção diferencial de transformadores. Kasztenny et al. (1997) apresentaram um algoritmo baseado em lógica nebulosa com doze regras para discriminar as situações de defeito de outras condições operativas. Já os autores Shin et al. (2003) propuseram um sistema nebuloso com restrições harmônicas e fluxo diferencial da corrente com objetivo de melhorar o desempenho do sistema de proteção nos casos de energização com baixo conteúdo de segundo harmônico e defeitos internos com altos componentes harmônicos de segunda ordem. Todavia, a maioria dessas abordagens não menciona o percentual do enrolamento que é protegido e não faz uma comparação com o método tradicional mais utilizado na proteção diferencial de transformadores (Harlow, 2006; Tripathy et al., 2005).

Neste contexto, este trabalho apresenta um algoritmo para a proteção diferencial de transformadores de potência baseado na lógica nebulosa, que permite um aprimoramento na análise das correntes passantes nestes equipamentos e, consequentemente, uma melhora no desempenho e um aumento no percentual do enrolamento que é protegido pelo sistema de proteção. Os testes da técnica proposta foram realizados com dados provenientes de um sistema elétrico modelado no software Alternative Transients Program (ATP), no qual diversas situações de operação e de defeito foram simuladas. O banco de dados formulado foi empregado para testar e validar a metodologia proposta, bem como para ensaios laboratoriais com um relé de proteção diferencial comercial.

\section{A PROTEÇÃO DIFERENCIAL}

A lógica diferencial é uma das principais metodologias aplicadas à proteção de transformadores de potência, cuja base é a comparação entre as correntes que entram 
e saem do equipamento, conforme ilustra a Figura 1. A figura mostra também o esquema de conexão dos Transformadores de Corrente (TCs) acoplados em série aos ramos primário e secundário. Neste, $N_{p}: N_{s}$ é a relação de transformação entre o primário e o secundário do transformador protegido.

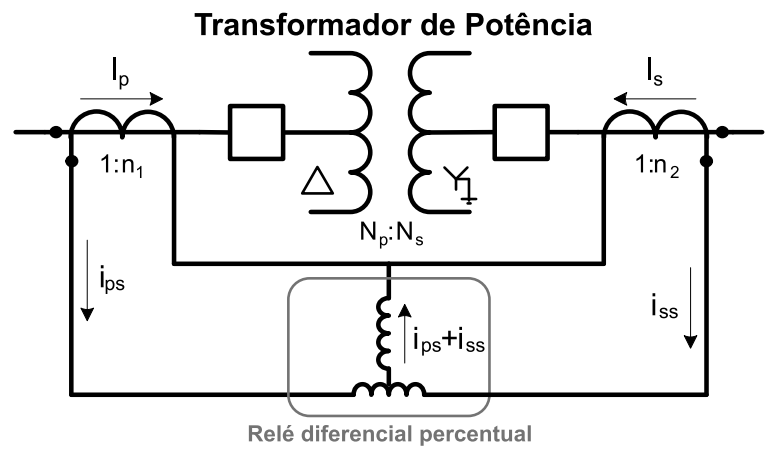

Figura 1: A proteção diferencial para transformadores.

Durante a operação normal do transformador e em caso de faltas externas, as correntes secundárias dos TCs registradas nos lados de alta $\left(i_{p s}\right)$ e de baixa tensão $\left(i_{s s}\right)$ do transformador protegido são iguais, desde que $N_{p} / N_{s}=n_{s} / n_{p}$, o que bloqueia a operação do relé diferencial. Todavia, durante os defeitos internos, a diferença entre as correntes será significativa, provocando a sensibilização do relé. Neste caso, a corrente diferencial (ou corrente de operação) pode ser obtida como a soma das correntes que entram no equipamento protegido (Segatto e Coury, 2008):

$$
i_{d i f}=\left|i_{p s}+i_{s s}\right|
$$

No entanto, existem várias fontes de erros que podem influenciar na sensibilidade do relé, como as relações de transformação dos TCs de proteção e as mudanças de tapes do transformador protegido, quando evidenciadas. Tal fato pode comprometer o funcionamento do sistema de proteção, proporcionando uma má operação do mesmo, pois pequenas correntes diferenciais podem ser caracterizadas, mesmo em condições normais de operação. Para corrigir esses problemas, define-se um limiar mínimo de atuação $\left(I_{p k p}\right)$ e uma corrente de restrição, conforme as equações (2) e (3).

$$
\begin{gathered}
i_{d i f} \geq I_{p k p} \\
i_{d i f} \geq i_{r t}
\end{gathered}
$$

nas equações, $i_{r t}=k\left|i_{p s}-i_{s s}\right|$ é a corrente de restrição, $k$ é o fator de compensação (geralmente 1 ou 0,5 ) e |...| é o valor absoluto. Por estas equações, o relé enviará um sinal de abertura para o disjuntor quando a corrente de operação for maior que a corrente de restrição (Harlow, 2006).

A Figura 2 ilustra a característica operativa de um relé diferencial típico, incluindo as suas zonas de operação e de restrição, bem como o valor da corrente diferencial instantânea irrestrita $\left(I_{\text {dinst }}\right)$. Alguns ajustes de restrição também são apresentados na figura, com o objetivo de acomodar possíveis correntes diferenciais provocadas pelos erros anteriormente citados.

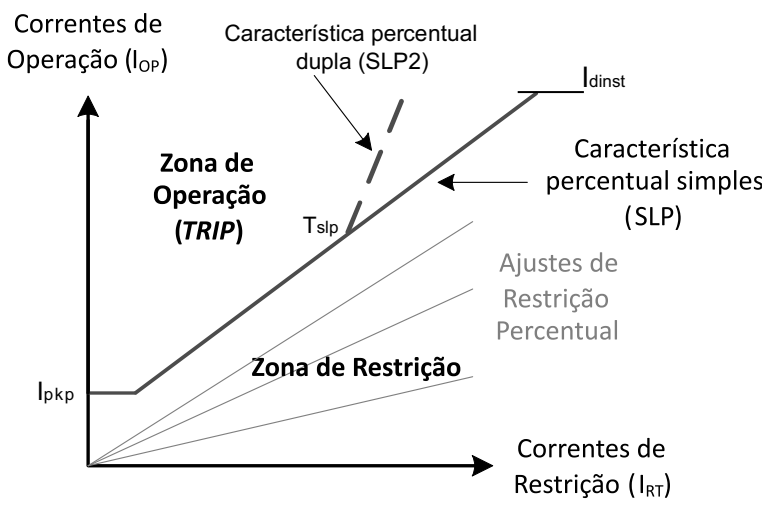

Figura 2: Curva característica da proteção diferencial percentual.

Cabe ressaltar que as falsas correntes diferenciais podem também surgir devido a algumas manobras realizadas no transformador e por situações de faltas próximas a este. Tais circunstâncias podem promover elevadas correntes diferenciais, suficientes para provocar uma atuação indevida da proteção diferencial. As principais causas para o surgimento dessas correntes indesejadas são listadas no que segue (Han et al., 2008; Wang et al., 2008; Bronzeado et al., 1996).

\subsection{Corrente de energização}

A corrente de energização, devido à magnetização e à saturação do núcleo do transformador quando do seu início de operação, tem a sua amplitude determinada pela inclinação da característica de magnetização na região saturada e pela indutância de escoamento do transformador.

Como fato, durante o procedimento de energização, o secundário do transformador permanece em aberto, o que causa o aparecimento de altas correntes diferenciais, as quais podem sensibilizar e operar indevidamente a proteção diferencial. 


\subsection{Sobreexcitação do transformador}

Durante a rejeição de carga e outras condições de operação, o transformador pode estar sujeito a sobretensões em regime permanente. Nestas condições, é observado um alto conteúdo harmônico de ordem ímpar inserido nas formas de onda coletadas, destacando-se as de $3^{a} \mathrm{e}$ $5^{a}$ ordem. Nessa conjuntura, o transformador apresenta características não lineares que causam o aparecimento de correntes diferenciais indesejadas e que podem provocar uma má atuação da proteção diferencial.

\subsection{Energização solidária}

A situação de energização solidária, ou sympathetic inrush, é geralmente tratada pela literatura assumindo que os transformadores estão isolados do sistema elétrico de potência (SEP), ou seja, sem que outros tipos de máquinas e/ou componentes elétricos estejam presentes no mesmo circuito. Na prática, porém, os equipamentos são energizados em paralelo com os demais dispositivos, o que pode provocar transitórios e sobretensões apreciáveis nos transformadores próximos, mesmo que estes já estejam em operação normal (Sengül et al., 2005; Kulkarni e Khaparde, 2004). Cabe ainda colocar que as correntes de energização evidenciadas na energização solidária possuem amplitudes superiores àquelas observadas quando ocorre a energização de um transformador que não opera em paralelo. A fim de melhor compreender como a energização de um transformador afeta as condições de funcionamento dos demais transformadores ligados na mesma barra, o sistema da Figura 3 é apresentado.

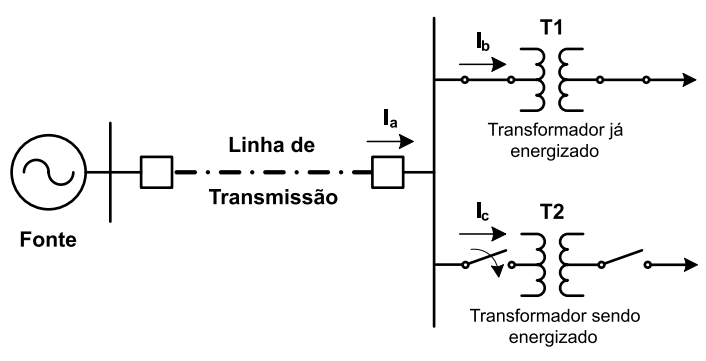

Figura 3: Sistema com energização solidária.

Ao conectar o transformador T2 à rede que já alimenta outros transformadores, como o transformador $T 1$, a corrente de energização produzida nesta conexão flui para os demais equipamentos e produz um fluxo em corrente contínua (CC) que se sobrepõe ao fluxo em corrente alternada (CA) normal de magnetização. Tal condição de operação aumenta a densidade de fluxo e proporciona altas correntes de magnetização no transformador vizinho $T 1$, mesmo sendo esta corrente solidária menor que a própria corrente de energização. Dependendo da amplitude do amortecimento da componente CC, o fenômeno de energização solidária pode elevar o nível de ruído emitido pelos transformadores devido ao aumento da densidade do fluxo no núcleo durante o período transitório (Kulkarni e Khaparde, 2004). É importante enfatizar que esta condição operativa pode provocar um mal funcionamento da proteção associada a este equipamento, já que correntes diferenciais indesejadas podem ser geradas (Barbosa et al., 2009).

\section{3 a lógica nebulosa e a PROTE- ÇÃO DIGITAL DIFERENCIAL IMPLE- MENTADA}

Cabe comentar que os sistemas nebulosos fazem parte, atualmente, de áreas de pesquisas conceituadas e consolidadas, sendo, por exemplo, amplamente utilizados em sistemas de controle e supervisão, onde, não se conhece, ou não se faz necessária a modelagem matemática do processo em análise.

A seguir, serão apresentadas as cinco etapas distintas consideradas na abordagem proposta pela aplicação da lógica nebulosa (Barbosa, 2010).

\subsection{Pré-processamento}

Para efeito de informação, na modelagem da filosofia diferencial proposta, todas as etapas referentes ao condicionamento do sinal, bem com ao sistema inteligente, foram contempladas e implementadas em linguagem de programação $\mathrm{C}++$. O fluxo das informações é realizado monofasicamente, ou seja, todos os procedimentos de pré-processamento e do sistema nebuloso associado são realizados para cada uma das três fases individualmente.

As tensões e as correntes trifásicas utilizadas pela metodologia desenvolvida são obtidas e condicionadas de forma similar aos equipamentos comerciais, o que permite uma melhor representação do processo de aquisição de dados. Desta forma, foram incluídas as etapas da filtragem anti-aliasing, a reamostragem, a correção da defasagem angular, a correção das relações de transformação dos TCs e a eliminação da sequência zero (IEEE Std. C37.91, 2008), conforme apresentado na Figura 4.

A aquisição de dados é realizada de forma janelada e com passo fixo de uma amostra. Desta forma, a execução do algoritmo será realizada sobre uma janela de dados contendo 16 amostras, respeitando-se o tempo hábil disponível para o processamento, que será quantificado pela disponibilização de uma nova amostra. A Figura 


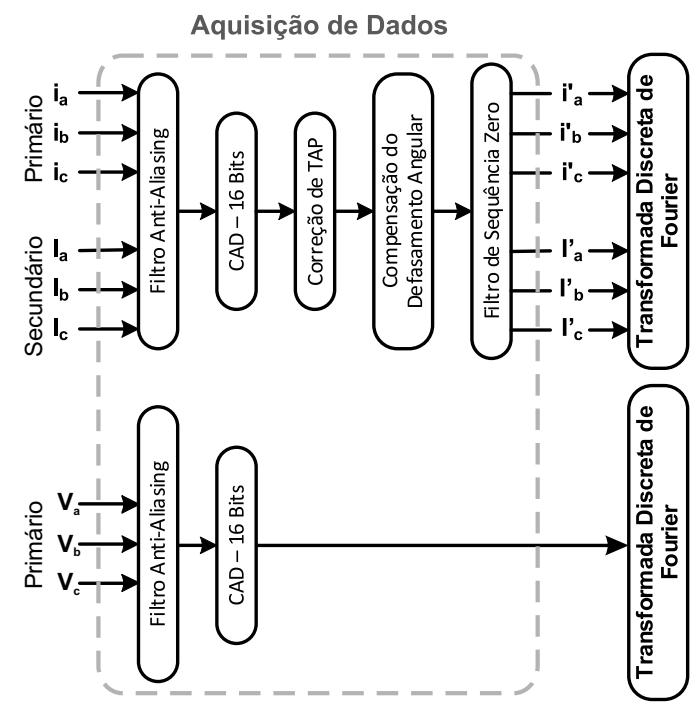

Figura 4: Diagrama do pré-processamento considerado.

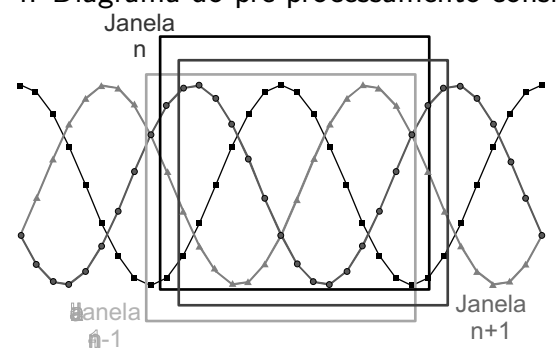

Figura 5: Progressão da janela de dados a cada amostra nova.

5 ilustra o movimento da janela de dados a cada nova amostra disponibilizada.

Após o condicionamento dos sinais, estes são submetidos à Transformada Discreta de Fourier (TDF) para a extração das componentes fundamentais e harmônicas, tanto das tensões e correntes primárias, quanto das correntes secundárias do transformador protegido. É importante observar que estes componentes serão utilizados no processo de discriminação das condições operativas do equipamento, uma vez que cada situação possui uma assinatura particular (Harlow, 2006).

\subsection{Fuzzificação}

Nesta etapa é avaliada a compatibilidade do dado de entrada com os conjuntos nebulosos correspondentes às variáveis de entrada, sendo as incertezas dos sinais de entrada quantificadas sem perdas da informação durante o processo. Desta forma, a fidelidade à qualidade dos dados de entrada do sistema inteligente depende diretamente dos conjuntos nebulosos de entrada e de saída.

As variáveis de entrada empregadas no sistema nebuloso foram:
1. $I_{o p}, 2 h$ e $5 h:$ Amplitude da corrente de operação fundamental $\left(I_{o p}=I_{d i f} / I_{r t}\right)$ e a maior amplitude das componentes harmônicas de $2^{a}$ e $5^{a}$ ordem das correntes do primário ou secundário do transformador protegido, respectivamente.

$$
\text { 2. } d_{F l x}=\frac{\frac{\Delta t}{2}\left(v_{p, k}-v_{p, k-1}\right)-L_{p}\left(i_{p, k}-i_{p, k-1}\right)}{\left(i_{p, k}-i_{s, k}\right)-\left(i_{p, k-1}-i_{s, k-1}\right)} \text { : }
$$

Amplitude estimada do fluxo magnético, na qual $p$ e $s$ representam o lado primário e secundário do transformador de potência, $\Delta t$ é o intervalo de amostragem, $i$ é a corrente que entra no equipamento, $v$ é a tensão medida nos terminais de entrada, $L_{p}$ é a indutância de dispersão do enrolamento primário e $k$ é o número da amostra (Phadke e Thorp, 1983).

A lógica nebulosa proposta utiliza a fuzzificação das quatro variáveis de entrada apresentadas mediante duas funções de pertinência do tipo trapezoidal, cujos ajustes foram baseados em informações encontradas na literatura (Kasztenny et al., 1997), em testes realizados e também com o conhecimento especialista. Estas funções de pertinência indicam se o valor da variável de entrada está "Baixo" ou "Alto", o que possibilitará a verificação do estado operacional do equipamento protegido, seja este de defeito e/ou de operação normal.

Os universos de discurso das variáveis de entrada foram definidos entre 0 (zero) e 1 (um) e descrevem o percentual do conteúdo harmônico $(2 h$ e $5 h)$ presente na janela analisada. Já a corrente de operação $\left(I_{o p}\right)$ e o fluxo magnético $\left(d_{F l x}\right)$ tiveram seus universos de discurso apontados entre 0 (zero) e 5 (cinco) por meio da análise de um conjunto de simulações computacionais pertinente ao contexto delineado. O intervalo entre 0 (zero) e 1 (um) também foi utilizado para a variável de saída para indicar as condições de operação normal e de defeito do equipamento a ser protegido. É factível salientar que para todas as situações operativas analisadas, não foram observados erros de operação do sistema nebuloso proposto ao limitar as variáveis utilizadas aos referidos intervalos. A Figura 6 ilustra os conjuntos de entrada e saída do sistema nebuloso considerado.

É importante destacar que apesar da semelhança na definição das variáveis de entrada entre o trabalho proposto e o algoritmo apresentado por Shin et al. (2003), existem diferenças nos tratamentos realizados nestas variáveis. Os autores supracitados verificam qual a amostra da janela analisada ultrapassou determinados limiares e utilizam esta informação no sistema nebuloso. Já no 


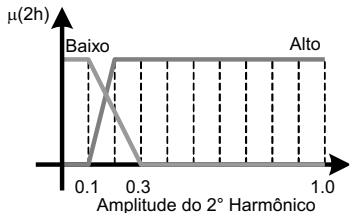

(a)

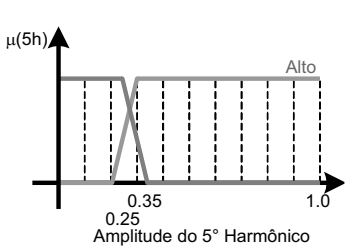

(c)

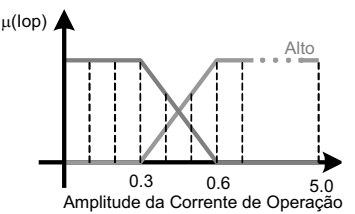

(b)

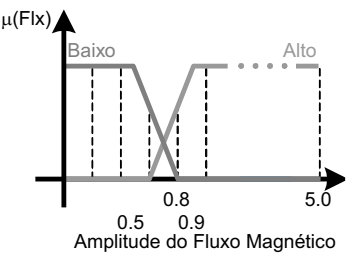

(d)

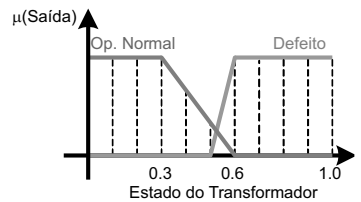

(e)

Figura 6: Funções de pertinência: (a) conjunto nebuloso de entrada 2h; (b) conjunto nebuloso de entrada $I_{o p}$; (c) Conjunto nebuloso de entrada $5 \mathrm{~h}$; e (d) conjunto nebuloso de entrada Flx. (e) Conjunto nebuloso de saída.

trabalho apresentado, não se aplica nenhum tipo de verificação prévia das variáveis em questão.

Apesar da incerteza sobre o conteúdo harmônico refletido nas correntes, as entradas apresentadas exprimem as características básicas para a solução do problema. Vale comentar que em situações de correntes diferenciais indesejadas e de defeito, as formas de onda distorcidas podem provocar erros na análise de Fourier utilizada, acarretando em erros na determinação dos parâmetros de entrada. Assim, a fuzzificação desses componentes permite uma melhor avaliação do fenômeno que está sendo observado.

\subsection{Métodos de inferência}

A etapa de inferência é responsável pelo mapeamento do conhecimento entre as entradas do sistema inteligente e sua respectiva saída por meio de um número limitado de regras nebulosas do tipo "SE-ENTÃO", conforme ilustrado a seguir:

SE o $2^{\circ}$ harmônico da corrente do primário (2h) é Baixo $\mathbf{E}$ o $5^{\circ}$ harmônico da corrente do primário (5h) é Baixo $\mathbf{E}$ a corrente de operação (Iop) é Baixa $\mathbf{E}$ o fluxo (Flx) é Baixo, Então a saída é Operação Normal

Neste contexto, o relé diferencial proposto utiliza 16 regras de inferência nebulosas, definidas por meio de co- nhecimento especialista, que relacionam as quatro entradas com uma respectiva saída por meio da regra de inferência composicional (ou modus ponens generalizado) em conjunto com o operador de implicação "min". A Tabela 1 apresenta as regras nebulosas utilizadas para a metodologia proposta.

Tabela 1: Resumo das regras de inferência nebulosas adotadas.

\begin{tabular}{cccccc}
\hline Regra & 2h & $\mathbf{5 h}$ & Iop & Flx & Saída \\
\hline \hline 1 & Baixo & Baixo & Baixo & Baixo & Op Normal \\
2 & Baixo & Baixo & Baixo & Alto & Op Normal \\
3 & Baixo & Baixo & Alto & Baixo & Defeito \\
4 & Baixo & Baixo & Alto & Alto & Defeito \\
5 & Baixo & Alto & Baixo & Baixo & Op Normal \\
6 & Baixo & Alto & Baixo & Alto & Op Normal \\
7 & Baixo & Alto & Alto & Baixo & Op Normal \\
8 & Baixo & Alto & Alto & Alto & Defeito \\
9 & Alto & Baixo & Baixo & Baixo & Op Normal \\
10 & Alto & Baixo & Baixo & Alto & Op Normal \\
11 & Alto & Baixo & Alto & Baixo & Op Normal \\
12 & Alto & Baixo & Alto & Alto & Defeito \\
13 & Alto & Alto & Baixo & Baixo & Op Normal \\
14 & Alto & Alto & Baixo & Alto & Op Normal \\
15 & Alto & Alto & Alto & Baixo & Op Normal \\
16 & Alto & Alto & Alto & Alto & Op Normal \\
\hline
\end{tabular}

Outro ponto a ser destacado em relação à proposta dos pesquisadores Shin et al. (2003) é a utilização integrada de todas as quatro variáveis de entrada para a formulação do processo de inferência, enquanto que no trabalho supracitado as entradas são processadas aos pares.

\subsection{Defuzzificação}

O procedimento de defuzzificação informa o valor numérico de saída do sistema nebuloso, o qual será utilizado para determinar o sinal de abertura (trip) ou de bloqueio do disjuntor. Utilizou-se a técnica do centro de área para extrair tal informação, sendo esta dada por (Kovacic e Bogdan, 2005):

$$
C D A=\frac{\sum_{k=1}^{N} \mu_{c}\left(V_{k}\right) V_{k}}{\sum_{k=1}^{N} \mu_{c}\left(V_{k}\right)}
$$

na qual, $N$ é o número de regras ativas e $\mu_{c}$ é o valor da função de pertinência no ponto desejado $\left(V_{k}\right)$. É importante salientar que este trabalho utilizou a biblioteca FisPro, desenvolvida em "C++", para a execução do sistema nebuloso (Guillaume et al., 2002).

\subsection{Tomada de decisão}

O sinal de trip é baseado na verificação do valor monofásico de saída do sistema nebuloso em relação a um limiar de bloqueio pré-estabelecido, bem como na confirmação desta condição por três vezes consecutivas. Isto é, se a saída nebulosa for maior que 0,5 , um contador (ctr_k) será incrementado e, quando este for igual a 3, com base 
em três respostas consecutivas, o relé enviará um sinal de abertura para os disjuntores associados. É importante enfatizar que a tomada de decisão, bem como o contador, são processados monofasicamente.

A Figura 7 mostra o diagrama de funcionamento básico do relé proposto.

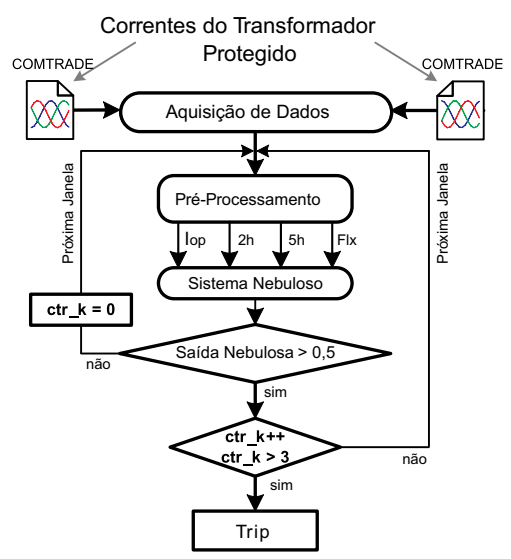

Figura 7: Diagrama básico do relé implementado.

\section{SISTEMA ELÉTRICO SIMULADO}

A Figura 8 mostra a representação do sistema elétrico utilizado nas simulações das condições operativas dos transformadores para avaliar o comportamento da proteção diferencial proposta.

O sistema elétrico é composto por um gerador síncrono de $13,8 \mathrm{kV}(60 \mathrm{~Hz})$ e de potência aparente de 90MVA, um motor de indução trifásico de $4,0 \mathrm{kV}$ e de potência de 1582HP, com seu respectivo transformador abaixador com potência aparente de $2 \mathrm{MVA}$ e relação de $13,8 / 4 \mathrm{kV}$, transformadores elevadores com relações de $13,8 / 138 \mathrm{kV}$ e de potência aparente de 25MVA, considerando sua curva de saturação, linhas de transmissão com extensões variando entre 50 e $100 \mathrm{~km}$, transformadores abaixadores similares aos elevadores e cargas caracterizadas por fator de potência de 0,92 indutivo e potência aparente variando entre 5 e $25 \mathrm{MVA}$.

Além dos equipamentos apresentados na Figura 8, foram modelados os TCs e TPs levando em consideração suas curvas de saturação para a obtenção dos sinais de tensão e corrente em análise, o sistema de controle de velocidade dinâmico para sistemas hidráulicos (Vieira Filho, 1984) e o controle automático de tensão (AVR) (IEEE Std. 421.5, 2006; Boldea, 2006). Maiores informações sobre o sistema elétrico utilizado neste trabalho podem ser encontradas nas referências (Barbosa et al., 2008; Gibelli et al., 2009).

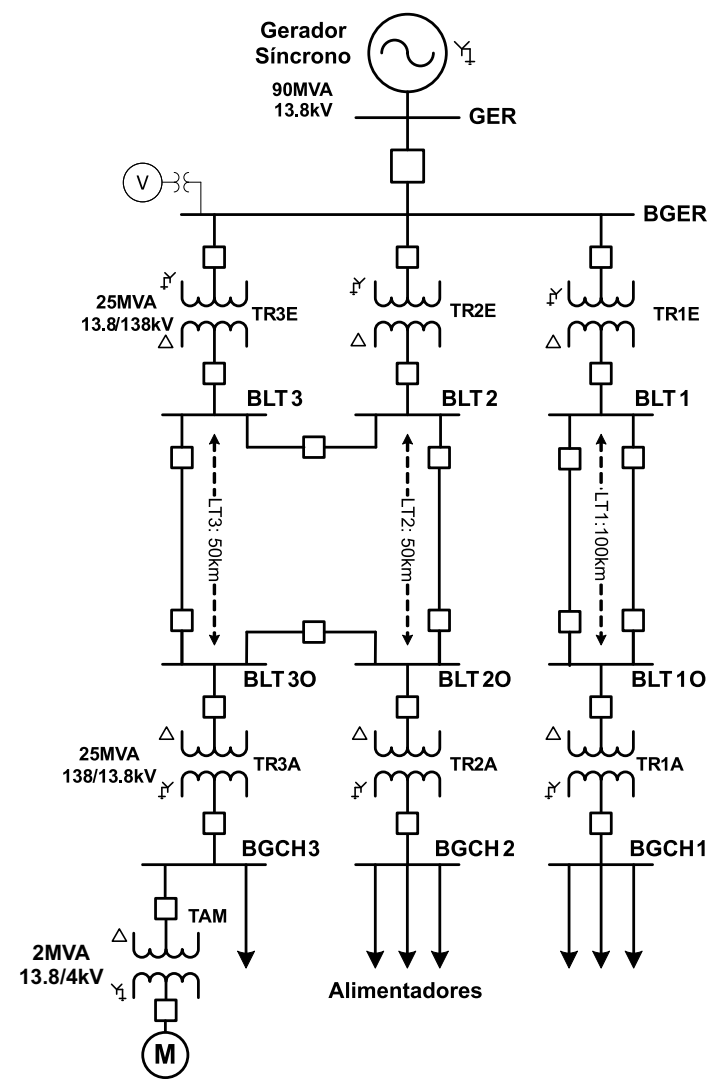

Figura 8: Representação do SEP analisado dispondo do software ATP.

\subsection{Geração dos casos de teste}

Os casos de teste foram gerados por meio de simulações provenientes do software ATP das diversas condições operativas dos transformadores de potência (Etapa A - Figura 9).

As condições consideradas foram:

- falta interna;

- falta entre espiras;

- energização;

- energização sob defeito,

- energização solidária; e

- sobre-excitação.

Após a obtenção das simulações, os arquivos resultantes foram convertidos para o COMTRADE (Common Format for Transient Data Exchange for Power Systems) (IEEE Std. C37.111, 1999) e inseridos em um banco de dados. Desse conjunto de dados, quinze casos foram 
selecionados para cada um dos seis cenários de teste anteriormente apresentados.

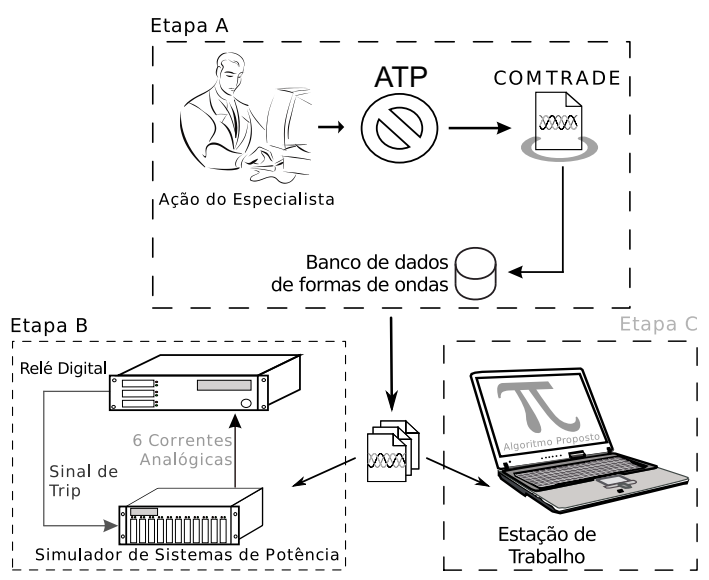

Figura 9: Esquema laboratorial desenvolvido.

A escolha dos casos em análise contemplou os ângulos de inserção (do defeito e/ou da energização) à $0^{\circ}, 45^{\circ} \mathrm{e}$ $90^{\circ}$ tendo a fase "A" como referência e as partições dos enrolamentos em $5 \%, 10 \%, 30 \%, 50 \%$ e $80 \%$ a partir do neutro para o enrolamento em estrela, e a partir da fase para o enrolamento em delta, permitindo uma melhor caracterização dos fenômenos. A Figura 10 ilustra as partições dos enrolamentos para a aplicação de faltas internas.
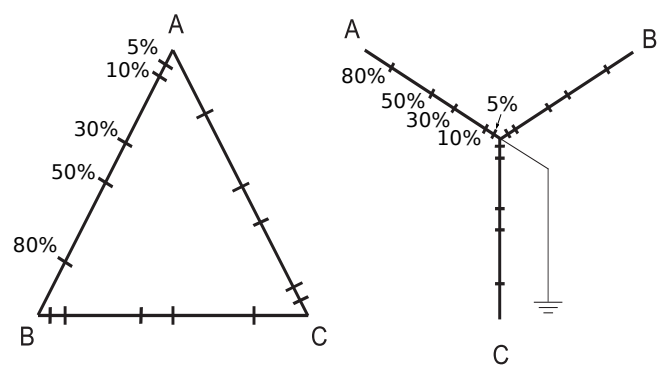

Figura 10: Modelagem do enrolamento para aplicação de faltas internas.

\section{ENSAIO LABORATORIAL}

A Figura 9 apresenta a metodologia de trabalho aplicada para o procedimento laboratorial proposto. Este arranjo experimental foi utilizado também para verificar o comportamento operativo da função $87 \mathrm{~T}$ implementada em um relé digital comercial, em relação aos diversos eventos simulados a partir do sistema elétrico modelado. A partir destes resultados será possível uma comparação real entre o relé comercial e a técnica proposta.

Os seguintes equipamentos foram utilizados neste procedimento: um simulador de sistemas de potência, um relé digital comercial e um microcomputador. Este úl- timo foi responsável pela coleta e análise das informações processadas.

A metodologia de trabalho é composta, basicamente, por três etapas distintas:

- Etapa A: modelagem e geração dos casos de interesse via aplicação do sofware ATP, bem como a formatação das situações de interesse para o COMTRADE;

- Etapa B: importação das situações de interesse pelo dispositivo simulador de sistemas de potência (caixa de teste) com a consequente aplicação dessas situações ao equipamento sob teste (relé comercial); e

- Etapa C: avaliação das respostas decorrentes.

O relé digital com a função $87 \mathrm{~T}$ utilizado nos ensaios laboratoriais possui dois slopes na característica diferencial percentual, conforme ilustrado pela Figura 2 (função de sobrecorrente e esquema de proteção por terra restrito), além dos registros oscilográficos e de eventos. Na Tabela 2, apresentam-se os ajustes utilizados a fim de se verificar o desempenho da função diferencial.

Tabela 2: Ajustes do relé comercial diferencial.

\begin{tabular}{cccc}
\hline Parâmet. & Ajuste (Un.) & Parâmet. & Ajuste (Un.) \\
\hline \hline $\mathrm{RTC}_{p}$ & $400: 1$ & $\mathrm{RTC}_{s}$ & $40: 1$ \\
$\mathrm{I}_{p k p}$ & $0.3(\mathrm{p} . \mathrm{u})$. & $\mathrm{I}_{d_{\text {inst }}}$ & $8.0(\mathrm{p} . \mathrm{u})$. \\
$\mathrm{SLP}_{1}$ & $25(\%)$ & $\mathrm{SLP}_{2}$ & $50(\%)$ \\
$\mathrm{I}_{b l}^{2 h}$ & $15(\%)$ & $\mathrm{I}_{b l}^{5 h}$ & $35(\%)$ \\
$\mathrm{REF}$ & off & $\mathrm{I}_{\text {inst }}$ & off \\
$\mathrm{T}_{s l p}$ & 3.0 (p.u.) & & \\
\hline
\end{tabular}

Na Tabela 2, $\mathrm{RTC}_{p}$ e $\mathrm{RTC}_{s}$ representam a relação de transformação dos TCs do lado primário e secundário do transformador de potência, $\mathrm{I}_{p k p}$ é o valor de pickup (de sensibilização) do elemento diferencial, $\mathrm{T}_{s l p}$ é o ponto de transição entre os dois slopes, $\mathrm{I}_{b l}^{2 h}$ e $\mathrm{I}_{b l}^{5 h}$ são as restrições de segunda e quinta harmônica, respectivamente, $R E F$ é a proteção de terra restrito, $I_{d_{i n s t}}$ é o ajuste da corrente diferencial irrestrita, $\mathrm{SLP}_{1}$ e $\mathrm{SLP}_{2}$ são os ajustes do primeiro e segundo slope e $\mathrm{I}_{\text {inst }}$ é o ajuste do elemento instantâneo de sobrecorrente.

\section{ANÁLISE DOS RESULTADOS}

O objetivo dessa seção é apresentar os resultados da abordagem proposta em comparação às obtidas por um relé disponível comercialmente.

Cabe afirmar que cada conjunto de testes foi repetido dez vezes e equações estatísticas foram utilizadas para 
determinar o tempo de operação do relé comercial testado (função $87 \mathrm{~T}$ ) e da técnica proposta. Todos os testes foram realizados com o objetivo de estabelecer um ponto de comparação entre o algoritmo desenvolvido e a técnica tradicional usualmente aplicada para a proteção destes equipamentos.

\subsection{Comparação de desempenho entre o relé comercial e a técnica proposta}

A Figura 11 mostra: (a) a forma de onda das correntes em ambos os lados do transformador protegido, (b) os canais digitais do relé comercial e (c) a saída nebulosa da técnica proposta para uma situação de energização, com ângulo de incidência à $0^{\circ}$ na fase "A", sob defeito interno em $10 \%$ do enrolamento da fase "A" no lado em estrela do transformador TR2E.

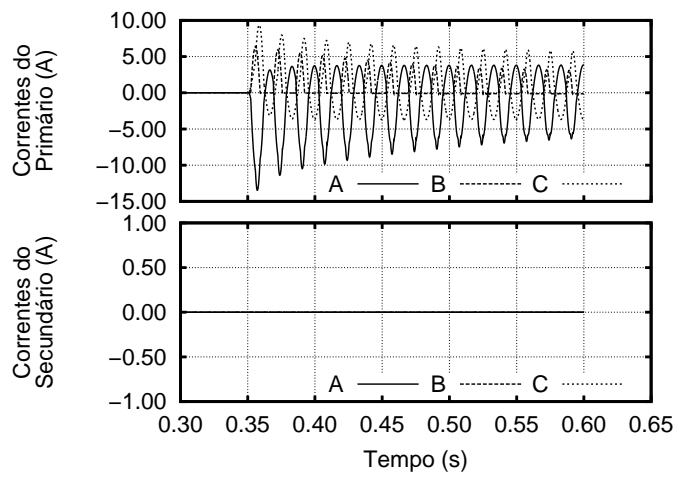

(a) Correntes do transformador.

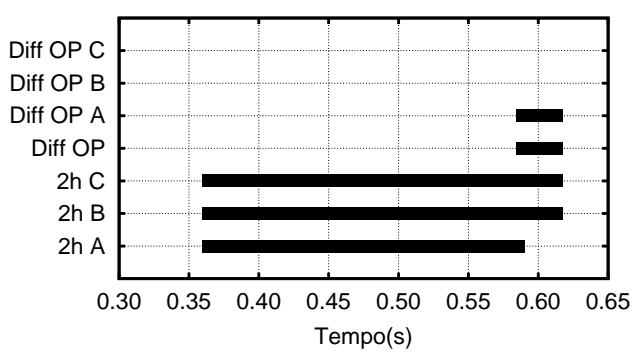

(b) Canais digitais do relé comercial $\left(t_{\text {trip }}=\right.$ $234,60 \mathrm{~ms}$ ).

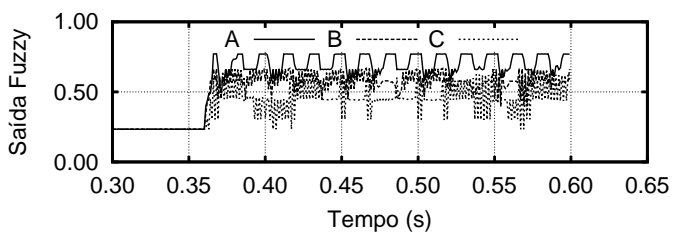

(c) Saída do algoritmo proposto $\left(t_{\text {trip }}=21,27 \mathrm{~ms}\right)$.

Figura 11: Energização sob defeito à $10 \%$ da fase "A" no enrolamento estrela.
Pela Figura 11 (b), observa-se, através dos canais digitais da oscilografia provenientes do relé comercial, um atraso de aproximadamente $210 \mathrm{~ms}$ para a sua atuação, provocado pelo bloqueio devido às restrições harmônicas. Tal atraso decorre da utilização de limiares préestabelecidos na comparação das componentes harmônicas. Contudo, tal tempo de atraso não ocorre no uso do sistema nebuloso proposto, pois como pode ser observado na Figura 11(c), este permitiu uma atuação eficiente do sistema de proteção em apenas $21,27 \mathrm{~ms}$.

Durante a realização dos testes, foi verificado que, nas situações avaliadas de energização com defeito próximo ao neutro, o algoritmo nebuloso foi mais rápido que o relé comercial. Isto é verdadeiro mesmo considerando a necessidade das três respostas consecutivas maiores do que 0,5 provenientes da saída do sistema nebuloso.

Outra observação interessante é que nos testes de energização sob defeito, o relé comercial (função 87T) utilizado não foi capaz de detectar defeitos inseridos a $5 \%$ do enrolamento estrela, próximo do neutro, conforme apresenta a Figura 12(b). Entretanto, enfatiza-se que para estas situações de defeitos próximos ao final do enrolamento estrela, o fabricante do equipamento recomenda a utilização da proteção de terra restrito. Contudo, tal função, por sua vez, aumenta a sensibilidade do esquema de proteção, tornando-a susceptível a atuações indevidas.

Por este cenário, foi verificada a robustez e a precisão do algoritmo proposto, uma vez que este detectou o defeito em aproximadamente $19 \mathrm{~ms}$ sem a utilização de funções complementares, conforme apresenta a Figura 12(c).

As Tabelas 3 e 4 apresentam o tempo de operação da técnica proposta e do relé comercial testado, respectivamente, para as 90 situações testadas. Os resultados apresentam informações das seis condições operativas simuladas, incluindo o tempo de operação máximo (MaxT) e mínimo (MinT), o tempo de operação médio $(\operatorname{AvrgT})$ e o desvio padrão do tempo de operação (DevT) para os casos de testes repetidos (reapresentados) por dez vezes.

Tabela 3: Resumo estatístico dos testes para o algoritmo proposto.

\begin{tabular}{cccccc}
\hline $\begin{array}{c}\text { Descrição da } \\
\text { condição } \\
\text { operativa }\end{array}$ & $\begin{array}{c}\text { AvrgT } \\
(\mathrm{ms})\end{array}$ & $\begin{array}{c}\text { MaxT } \\
(\mathrm{ms})\end{array}$ & $\begin{array}{c}\text { MinT } \\
(\mathrm{ms})\end{array}$ & $\begin{array}{c}\text { DevT } \\
(\mathrm{ms})\end{array}$ & $\begin{array}{c}\text { Erro } \\
(\%)\end{array}$ \\
\hline \hline Energização & - & - & - & - & 0 \\
Energ. sob defeito & 18,57 & 21,87 & 15,49 & 1,46 & 0 \\
Energ. solidária & - & - & - & - & 0 \\
Falta interna (AT) & 13,57 & 25,66 & 7,43 & 4,81 & 0 \\
Falta entre espiras & 12,12 & 18,04 & 8,35 & 2,44 & 0 \\
Sobre-excitação & - & - & - & - & 0 \\
\hline
\end{tabular}




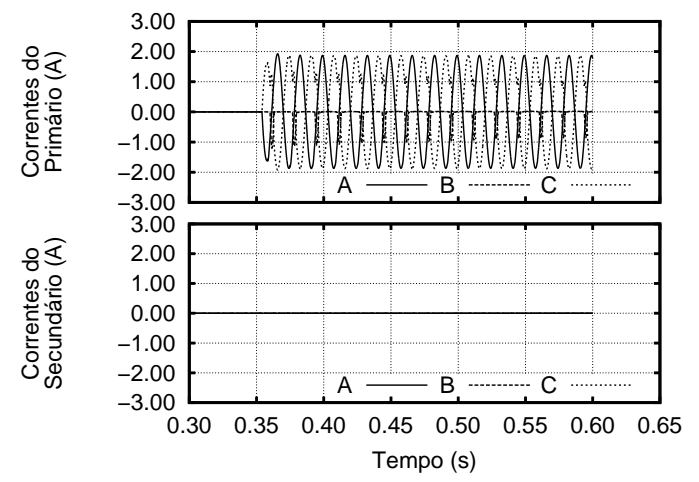

(a) Correntes do transformador.

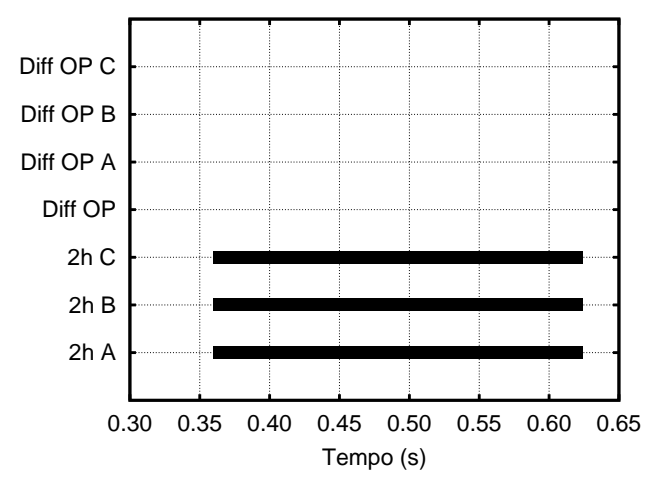

(b) Canais digitais do relé comercial (Sem trip).

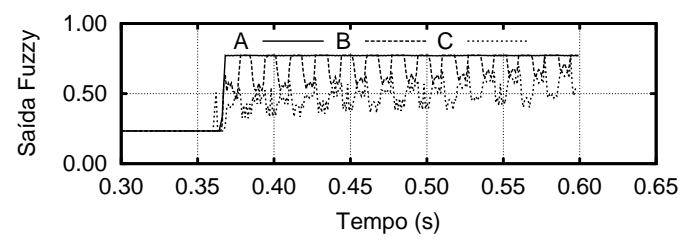

(c) Saída do algoritmo proposto $\left(t_{\text {trip }}=19,10 \mathrm{~ms}\right)$.

Figura 12: Energização sob defeito à $5 \%$ da fase "A" no enrolamento estrela.

É importante observar que os erros relativos ao equipamento comercial estão associados a defeitos próximos ao final do enrolamento, ou seja, que afetam menos que $10 \%$ do enrolamento estrela. A Figura 13 exemplifica uma dessas situações, na qual o transformador protegido sofre com um defeito a $5 \%$ do enrolamento estrela próximo do neutro. Destaca-se, neste caso, que existe uma pequena alteração no comportamento das correntes do transformador defeituoso, o que implica em uma grande dificuldade na detecção deste.

Como apresentado, observa-se que para as situações de energização, energização solidária e de sobreexcitação, tanto o relé comercial como a técnica proposta apresentaram as respostas desejadas, ou seja, não foram sensibilizados por estas ocorrências.
Tabela 4: Resumo estatístico dos testes para o relé comercial.

\begin{tabular}{cccccc}
\hline $\begin{array}{c}\text { Descrição da } \\
\text { condição } \\
\text { operativa }\end{array}$ & $\begin{array}{c}\text { AvrgT } \\
(\mathrm{ms})\end{array}$ & $\begin{array}{c}\text { MaxT } \\
(\mathrm{ms})\end{array}$ & $\begin{array}{c}\text { MinT } \\
(\mathrm{ms})\end{array}$ & $\begin{array}{c}\text { DevT } \\
(\mathrm{ms})\end{array}$ & $\begin{array}{c}\text { Erro } \\
(\%)\end{array}$ \\
\hline \hline Energização & - & - & - & - & 0 \\
Energ. sob defeito & 42,08 & 240,70 & 21,50 & 49,75 & 20 \\
Energ. solidária & - & - & - & - & 0 \\
Falta interna (AT) & 24,34 & 27,00 & 22,00 & 1,34 & 20 \\
Falta entre espiras & 24,08 & 26,80 & 21,30 & 1,33 & 0 \\
Sobre-excitação & - & - & - & - & 0 \\
\hline
\end{tabular}

\section{CONCLUSÕES}

Este trabalho apresentou uma técnica alternativa para a proteção diferencial digital de transformadores de potência utilizando a lógica nebulosa. Um conjunto de regras de inferência e subrotinas baseadas na lógica nebulosa foram desenvolvidas em linguagem de programação $\mathrm{C}++$ com o intuito de identificar defeitos internos e discriminá-los de outras situações operativas. A metodologia proposta baseou-se nas restrições harmônicas, na corrente de operação e na variação de fluxo magnético no núcleo, incluindo limiares de atuação.

Após as análises e comparações dos resultados, foi possível observar as seguintes vantagens do algoritmo desenvolvido em relação ao relé comercial e a outros algoritmos que utilizam a lógica nebulosa:

1. Aumento do percentual do enrolamento protegido quando comparado a equipamentos comerciais, uma vez que esta informação não é apresentada na maioria dos trabalhos encontrados na literatura;

2. A tomada de decisão se mostrou robusta e confiável, uma vez que em situações de ocorrência de falha do equipamento comercial, o algoritmo nebuloso atuou corretamente;

3. O tempo de atuação do algoritmo nebuloso foi menor do que o requerido pelo equipamento comercial para produzir os resultados;

4. O algoritmo nebuloso pode ser aplicado às diversas situações e equipamentos, uma vez que as regras de inferência e as entradas do processo de fuzzificação são flexíveis e independem das características construtivas do transformador;

5. A decisão de trip foi baseada em uma simples comparação, reduzindo a ação de abertura do disjuntor à lógica booleana;

6. Facilidade de aplicação do algoritmo nebuloso, já que a configuração é única, não necessitando de ajustes adicionais e conhecimento das demais funções do relé, como no caso do relé 87 utilizado; 


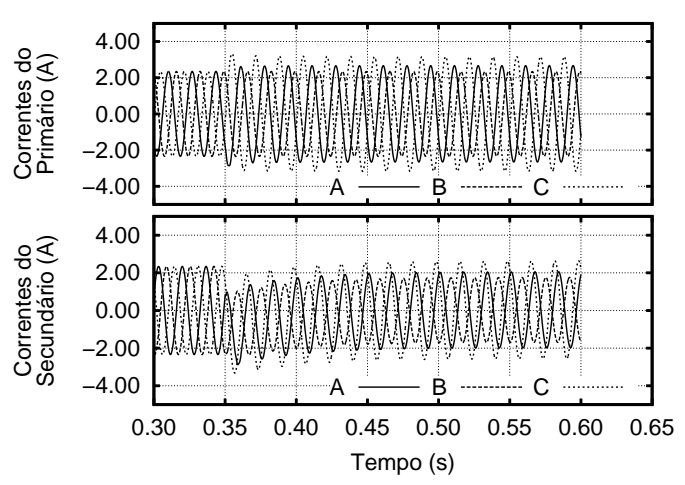

(a) Correntes do transformador.

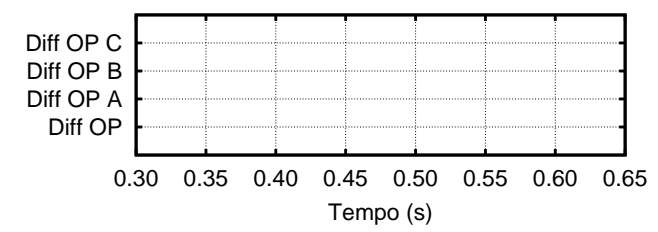

(b) Canais digitais do relé comercial (Sem trip).

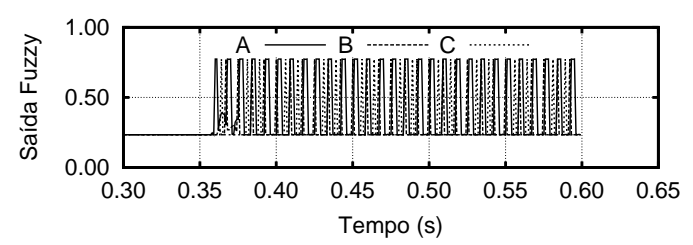

(c) Saída do algoritmo proposto $\left(t_{\text {trip }}=19,10 \mathrm{~ms}\right)$.

Figura 13: Defeito interno aplicado à 90 graus, em $5 \%$ do enrolamento, considerando a fase $A$ na conexão em estrela.

7. Supervisão da decisão de trip, a partir do contador evidenciado na Figura 7, o que fornece maior confiabilidade ao algoritmo;

8. Não utiliza a característica diferencial percentual como variável de entrada, simplificando o processo de ajuste do algoritmo;

9. Maior sensibilidade do sistema nebuloso utilizado, pois o limiar de atuação da saída nebulosa é igual a 0,5 .

Embora o algoritmo proposto possua vantagens em relação ao equipamento comercial existem algumas ressalvas em sua aplicação, como a utilização das tensões primárias e a utilização da indutância de dispersão do primário do transformador.

Outro aspecto importante é que o relé comercial apresentou menor desvio padrão nos tempos de atuação para as condições de falta interna e para faltas entre espiras, o que implica em dizer que a técnica tradicional nele contida apresenta uma maior constância em rela- ção ao algoritmo proposto. Contudo, para as situações de energização sob defeito avaliadas, houve uma grande discrepância entre os desvios observados, em favor do algoritmo proposto.

\section{AGRADECIMENTOS}

Os autores gostariam de agradecer ao Laboratório de Sistemas de Energia Elétrica (LSEE), da Escola de Engenharia de São Carlos (EESC-USP) pela infraestrutura proporcionada, assim como à Fundação de Amparo à Pesquisa do Estado de São Paulo (FAPESP) e à Coordenação de Aperfeiçoamento de Pessoal de Nível Superior (CAPES) pelo suporte financeiro disponibilizado para a realização desta pesquisa.

\section{REFERÊNCIAS}

Barbosa, D. (2010). Sistema Híbrido Inteligente para o Monitoramento e Proteção de Transformadores de Potência, PhD thesis, Escola de Engenharia de São Carlos da Universidade de São Paulo, São Carlos SP.

Barbosa, D., Monaro, R. M., Coury, D. V. e Oleskovicz, M. (2008). Filtragem adaptativa para a estimação da frequência em sistemas elétricos de potência, SBA Controle $\&$ Automação 19(2): 226-234.

Barbosa, D., Netto, U. C., Branco, H. M. G. C., Coury, D. V. e Oleskovicz, M. (2009). Impacto do paralelismo na proteção diferencial de transformadores de potência, 8th Latin-American Congress on Electricity Generation and Transmission, Ubatuba.

Blackburn, J. L. e Domin, T. J. (2007). Protective Relaying Principles and Applications, 3 edn, CRC Press, Boca Raton.

Boldea, I. (2006). Synchronous Generators, CRC Press, Boca Raton. ISBN: 084935725X.

Bronzeado, H., Brogan, P. e Yacamini, R. (1996). Harmonic analysis of transient currents during sympathetic interaction, IEEE Transactions on Power Systems 11(4): 2051-2056.

Gibelli, G. B., Chemin Netto, U., Barbosa, D., Oleskovicz, M. e Coury, D. V. (2009). Suportabilidade e o desempenho da operação dos motores de indução trifásicos frente aos afundamentos de tensão, VIII Conferência Brasileira sobre Qualidade da Energia Elétrica (CBQEE), Blumenau - Brasil. 
Guillaume, S., Charnomordic, B. e Lablée, J.-L. (2002). FisPro: An open source portable software for fuzzy inference systems, INRA-Cemagref.

Han, Z., Liu, S., Gao, S. e Bo, Z. (2008). Flow-through inrush and its effect on transformer differential protection, Proc. IET 9th International Conference on Developments in Power System Protection DPSP 2008, pp. 296-301.

Harlow, J. H. (ed.) (2006). Electric Power Engineering Handbook, CRC Press. ISBN: 9780849392931.

IEEE Std. 421.5 (2006). IEEE recommended practice for excitation system models for power system stability studies, IEEE Std 421.5-2005 (Revision of IEEE Std 421.5-1992) pp. 1-85.

IEEE Std. C37.111 (1999). IEEE standard common format for transient data exchange (comtrade) for power systems.

IEEE Std. C37.91 (2008). IEEE guide for protecting power transformers. ISBN: 978-0-7381-5389-6.

Kasztenny, B., Rosolowski, E., Saha, M. e Hillstrom, B. (1997). A self-organizing fuzzy logic based protective relay-an application to power transformer protection, IEEE Transactions on Power Delivery 12(3): 1119-1127.

Kovacic, Z. e Bogdan, S. (2005). Fuzzy Controller Design (Control Engineering (Taylor \& Francis)), CRC Press, Inc., Boca Raton, FL, USA.

Kulkarni, S. e Khaparde, S. (2004). Transformer Engineering: Design and Practice, Power Engineering (Willis), CRC Press, New York. ISBN: 9780824756536 .

Megahed, A. I., Ramadan, A. e El-Mahdy, W. (2008). Power transformer differential relay using wavelet transform energies, Proc. IEEE Power and Energy Society General Meeting - Conversion and Delivery of Electrical Energy in the 21st Century, pp. 1-6.

Perez, L. G., Flechsig, A. J., Meador, J. L. e Obradovic, Z. (1994). Training an artificial neural network to discriminate between magnetizing inrush and internal faults, IEEE Transactions on Power Delivery 9(1): 434-441.

Phadke, A. G. e Thorp, J. S. (1983). A new computer-based flux-restrained current-differential relay for power transformer protection, IEEE Transactions on Power Apparatus and Systems PAS102(11): 3624-3629.
Segatto, E. C. e Coury, D. (2008). Redes neurais aplicadas a relés diferenciais para transformadores de potência, SBA Controle \& A Automação 19(1): 93 106.

Sengül, M., Alboyaci, B. e Cetinkaya, H. B. (2005). Case study of symphatetic interaction between transformers caused by inrush transients, International Conference pn Power Systems Transients, number 125, Montreal, Canada.

Shin, M.-C., Park, C.-W. e Kim, J.-H. (2003). Fuzzy logic-based relaying for large power transformer protection, IEEE Transactions on Power Delivery 18(3): 718-724.

Tripathy, M., Maheshwari, R. P. e Verma, H. K. (2005). Advances in transform protection: A review, Electric Power Components and Systems 33(11): 1203 $-1209$.

Tripathy, M., Maheshwari, R. P. e Verma, H. K. (2006). Particle swarm optimization based probabilistic neural network for power transformer protection, Proc. IEEE International Conference on Industrial Technology ICIT 2006, pp. 568-573.

Tripathy, M., Maheshwari, R. P. e Verma, H. K. (2010). Power transformer differential protection based on optimal probabilistic neural network, IEEE Transactions on Power Delivery 25(1): 102-112.

Vieira Filho, X. (1984). Operation of Power System with Automatic Generation Control (Portuguese), Editora Campus Ltda, Rio de Janeiro.

Wang, Y., Yin, X., You, D. e Xu, T. (2008). Analysis on the influencing factors of transformer sympathetic inrush current, 2008 IEEE Power and Energy Society General Meeting - Conversion and Delivery of Electrical Energy in the 21st Century, pp. 1-8. 